\title{
Two-qubit quantum computing in a projected subspace
}

\author{
Bi Qiao, ${ }^{1,2,3}$ H. E. Ruda, ${ }^{1}$ and M. S. Zhan ${ }^{2}$ \\ ${ }^{1}$ Energenius Centre for Advanced Nanotechnology, University of Toronto, Toronto, Canada M5S 3E4 \\ ${ }^{2}$ Wuhan Institute of Physics and Mathematics, Chinese Academy of Science, Wuhan 430071, China \\ ${ }^{3}$ Complexity Science Center, Yangzhou University, Yangzhou 225002, China
}

(Received 5 September 2001; published 10 April 2002)

\begin{abstract}
A formulation for performing quantum computing in a projected subspace is presented, based on the subdynamical kinetic equation (SKE) for an open quantum system. The eigenvectors of the kinetic equation are shown to remain invariant before and after interaction with the environment. However, the eigenvalues in the projected subspace exhibit a type of phase shift to the evolutionary states. This phase shift does not destroy the decoherence-free (DF) property of the subspace because the associated fidelity is 1 . This permits a universal formalism to be presented-the eigenprojectors of the free part of the Hamiltonian for the system and bath may be used to construct a DF projected subspace based on the SKE. To eliminate possible phase or unitary errors induced by the change in the eigenvalues, a cancellation technique is proposed, using the adjustment of the coupling time, and applied to a two-qubit computing system. A general criteria for constructing a DF-projected subspace from the SKE is discussed. Finally, a proposal for using triangulation to realize a decoherence-free subsystem based on SKE is presented. The concrete formulation for a two-qubit model is given exactly. Our approach is general and appears to be applicable to any type of decoherence.
\end{abstract}

DOI: 10.1103/PhysRevA.65.042325

PACS number(s): 03.67.Lx, 03.65.Fd, 03.65.Yz, 89.70.+c

\section{INTRODUCTION}

Solid-state proposals for quantum computers have progressed markedly of late: these include superconducting junctions, quantum dots, electron spin resonance, nuclear spins of impurity atoms, and nuclear spins in a crystal lattice [1-5]. The working conditions for most proposals require low operating temperatures (typically a few kelvins). Ideally, if such a system can be created as isolated from the environment, system evolution for a given quantum-computing process, may be described by a unitary operator with timereversal symmetry. Often this is not the case for real quantum-computing systems because the interaction between such systems and their environment introduces decoherence, which destroys the superpositions of qubits that enable the quantum logical operations to be validated $[6,7]$. Such decoherence is a major obstacle in developing a practical quantum computer. Recent publications have formulated a theory for a decoherence-free (DF) subspace in which quantumcomputing is performed. The first formulation of DF conditions was performed by Zanardi and Rasetti [8,9]. This original formulation is quite general, which is not in the form of a master equation and does not invoke the Born-Markov approximation. Further work by Viola and Zanardi deal with DF subspaces by quantum-control theory in a nonMarkovian setting [10-12]. Then an important direction of the DF-subspace theory was developed from a master equation (such as the Lindblad equation) for the open system within the powerful semigroup approach [13-17]. A quantum jump description for the state of the atoms has also derived a consistent result with master equation for dipole interaction atoms, but may give more insight into the time evolution of a single system [18]. Experimentally, DF subspace have recently been observed, which shows that such DF subspaces do indeed exist, allowing logical qubits to be encoded without decoherence $[19,20]$. These provide the motivation for building appropriate open quantum-computing systems suitable for (a) canceling the effects of intrinsic decoherence, and thus, behaving as ideal quantum-computing systems in the subspaces, or; (b) construct DF subspace without invoking any approximations (such as the BornMarkov approximation) or restrictions on the type of decoherence (e.g., symmetric and collective decoherence). In this open system, self-adjoint operators and unitary-evolution groups are not intrinsically required for quantum computation. Quantum computation can then be performed in a more general functional space including triplet structures, rigged Hilbert space (RHS), $\Phi \subset \mathcal{H} \subset \Phi^{\times}$, or rigged Liouville space, $\Phi \otimes \Phi \subset \mathcal{H} \otimes \mathcal{H} \subset^{\times} \Phi \otimes \Phi^{\times}$, where $\mathcal{H}$ is Hilbert space, $\Phi$ is dense subspace of $\mathcal{H}$, and $\Phi^{\times}$is dual space of $\Phi$, rather than just Hilbert or Liouville space [21-23].

In this paper, we present a formulation for performing quantum computation in a DF projected subspace based on the subdynamical equation (SKE). In Sec. II, we briefly introduce a subdynamical formulation in the Hamiltonian representation. In Sec. III, we propose a type of DF-projected subspace. In Sec. IV and V, these concepts are applied to the example of a two-qubit spin-computing system plus Bosonic bath; a procedure to cancel the phase error induced by the change of eigenvalues is discussed. In Sec. VI, a general approach for obtaining the necessary and sufficient condition for DF behavior is discussed. In Sec. VII, a triangulating method is introduced for determining the DF-projected subspace exactly.

\section{SUBDYNAMICS FORMULATION}

Consider a quantum system that is composed of $N$ quantum registers and interacts with a large thermal reservoir. We denote $H_{S}(t), H_{B}$, and $H_{i n t}$ as the Hamiltonian of the system 
$S$, the Hamiltonian of the the thermal reservoir $B$, and the interaction between $S$ and $B$, respectively. Then the total Hamiltonian of the system plus the reservoir can be expressed as $H_{S}(t) \otimes I_{B}+I_{S} \otimes H_{B}+\lambda H_{i n t}$, with the corresponding Schrödinger equation as

$$
i \frac{\partial}{\partial t} \psi(t)=H(t) \psi(t) \text {. }
$$

If one chooses the time-independent eigenprojectors of $H_{S}(t) \otimes I_{B}+I_{S} \otimes H_{B}$ as $P_{\nu} \equiv\left|\varphi_{\nu}\right\rangle\left\langle\varphi_{\nu}\right|$ and $Q_{\nu}$ with $P_{\nu}+Q_{\nu}$ $=1$ and the eigenprojectors of the total Hamiltonian as $\Pi_{\nu}(t)$ to satisfy the Heisenberg equation, then, from the definition of $\Pi_{\nu}(t)$ one can induce the definition of the creation correlation operator $C_{\nu}(t)\left(D_{\nu}(t)\right) \quad$ as $\quad Q_{\nu} \Pi_{\nu}(t)$ $=C_{\nu}(t) \Pi_{\nu}(t)\left(\Pi_{\nu}(t) Q_{\nu}=\Pi_{\nu}(t) D_{\nu}(t)\right)$. Note that the operator $C_{\nu}(t)$ creates the $Q_{\nu}$ part of $\Pi_{\nu}(t)$ from $P_{\nu}$ and the operator $D_{\nu}(t)$ destroys the $Q_{\nu}$ part of $\Pi_{\nu}(t)$ from $P_{\nu}$, since $C_{\nu}(t)=Q_{\nu} C_{\nu}(t) P_{\nu}$ and $D_{\nu}(t)=P_{\nu} D_{\nu}(t) Q_{\nu}$. This enables a projected kinetic equation to be constructed in the projected subspace by

$$
i \frac{\partial}{\partial t} \sum_{\nu}\left[P_{\nu} \Pi_{\nu}(t) \psi(t)\right]=\Theta(t) \sum_{\nu}\left[P_{\nu} \Pi_{\nu}(t) \psi(t)\right],
$$

with an intermediate operator defined as

$$
\begin{aligned}
\Theta(t) & \equiv\left[H_{0}^{S}(t)+H_{0}^{B}+H_{0}^{i n t}\right]+\left[H_{1}^{S}(t)+H_{1}^{B}+H_{1}^{i n t}\right] C(t) \\
& \equiv \Theta_{0}(t)+H_{0}^{i n t}+\left[H_{1}^{S}(t)+H_{1}^{B}+H_{1}^{i n t}\right] C(t),
\end{aligned}
$$

here $H_{0}^{S, B, i n t}(t)$ and $H_{1}^{S, B, i n t}(t)$ are the diagonal and offdiagonal parts of the corresponding total Hamiltonian, respectively. $\Pi_{\nu}(t)$ can be found from the subdynamics formulation as $\left(P_{\nu}+C_{\nu}(t)\right)\left(P_{\nu}+D_{\nu}(t) C_{\nu}(t)\right)^{-1}\left(P_{\nu}+D_{\nu}(t)\right)$ $[21,22]$. The creation (destruction) operator can be obtained from the basic operator equations in the subdynamics formulation. Indeed, from the definition of the eigenprojectors $\Pi_{\nu}(t)$ we have

$$
\left[E_{\nu}(t)-Q_{\nu} H(t) Q_{\nu}\right] Q_{\nu} \Pi_{\nu}(t)=Q_{\nu} H_{i n t} P_{\nu} \Pi_{\nu}(t)
$$

and hence yielding the crucial relationship

$$
Q_{\nu} \Pi_{\nu}(t)=Q_{\nu} C(t) P_{\nu} \Pi_{\nu}(t),
$$

with definitions

$$
C(t) \equiv \sum_{\mu} \frac{1}{E_{\mu}(t)-Q_{\mu} H(t) Q_{\mu}} Q_{\mu} H(t) P_{\mu},
$$

and

$$
C_{\nu}(t) \equiv Q_{\nu} C(t) P_{\nu}=\frac{1}{E_{\nu}(t)-Q_{\nu} H(t) Q_{\nu}} Q_{\nu} H_{i n t} P_{\nu},
$$

where $E_{\nu}(t)$ is $\nu$ th eigenvalue of the total Hamiltonian $H(t)$. In the same way,

$$
D_{\nu}(t)=P_{\nu} H_{i n t} Q_{\nu} \frac{1}{E_{\nu}(t)-Q_{\nu} H(t) Q_{\nu}} .
$$

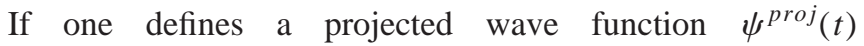
by $\psi(t) \equiv \Sigma_{\nu}\left[P_{\nu}+C_{\nu}(t)\right] \psi_{\nu}^{p r o j}(t)$, then $\Sigma_{\nu} P_{\nu} \Pi_{\nu}(t) \psi(t)$

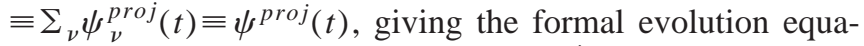
tion for a projected wave function $\psi^{p^{r o j}}(t)$ as

$$
\begin{aligned}
\psi^{p r o j}(t)= & \hat{T} \exp \left[-i \int_{t_{0}}^{t} d t^{\prime} \Theta\left(t^{\prime}\right)\right] \psi^{p r o j}(t)\left(t_{0}\right) \\
= & \hat{T} \exp \left[-i \int_{t_{0}}^{t} d t^{\prime} \Theta\left(t^{\prime}\right)\right] \sum_{\nu}\left[P_{\nu}+D_{\nu}\left(t_{0}\right)\right. \\
& \left.\times C_{\nu}\left(t_{0}\right)\right]^{-1}\left[P_{\nu}+D_{\nu}\left(t_{0}\right)\right] \psi\left(t_{0}\right)
\end{aligned}
$$

\section{DF PROJECT SUBSPACE}

Using the above subdynamics formulation, it is apparent that before the interaction occurs, the spectral decomposition for $\left[H_{S}(t)+H_{B}\right]$ is the same as that for the total intermediate operator $\Theta(t)$, i.e.,

$$
H_{S}(t)+H_{B}=\Theta(t)=\sum_{\nu} E_{\nu}^{0}(t) P_{\nu}
$$

where $E_{\nu}^{0}(t)$ is the $\nu$ th eigenvalue of $\left[H_{S}(t)+H_{B}\right]$. But once the interaction with the environment has occurred, the eigenvalues change from $E_{\nu}^{0}(t)$ to $E_{\nu}^{0}(t)+\triangle E_{\nu}(t)$, where the definition

$$
\triangle E_{\nu}(t)=\left\langle\phi_{\nu}\left|H_{1}^{i n t} C(t)\right| \phi_{\nu}\right\rangle
$$

is made. The corresponding eigenprojectors $P_{\nu}$ of $\Theta(t)$ still remain invariant. The spectral decomposition of the intermediate operator $\Theta(t)$, thus, is different from $\left(H_{S}(t)+H_{B}\right)$,

$$
\Theta(t)=\sum_{\nu}\left[E_{\nu}^{0}(t)+\triangle E_{\nu}(t)\right] P_{\nu}
$$

The corresponding mixed-state fidelity in the projected subspace can be calculated from 


$$
\begin{aligned}
& F(t)=\operatorname{Tr} \sqrt{\rho^{p r o j}\left(t_{0}\right) \rho^{p r o j}(t)}=\operatorname{Tr} \sqrt{\rho^{p r o j}\left(t_{0}\right) \hat{T} \exp \left[-i \int \Theta\left(t^{\prime}\right) d t^{\prime}\right] \rho^{p r o j}\left(t_{0}\right) \hat{T} \exp \left[i \int \Theta\left(t^{\prime}\right) d t^{\prime}\right]} \\
& =\operatorname{Tr} \sqrt{\left(\sum_{\nu, \mu}\left[\rho^{p r o j}\left(t_{0}\right)\right]_{\nu \mu} P_{\nu \mu}\right)}\left(\hat{T} \exp \left[-i \int \Theta\left(t^{\prime}\right) d t^{\prime}\right] \sum_{\nu, \mu}\left[\rho^{p r o j}\left(t_{0}\right)\right]_{\nu \mu} P_{\nu \mu} \hat{T} \exp i \int \Theta\left(t^{\prime}\right) d t^{\prime}\right)
\end{aligned}
$$

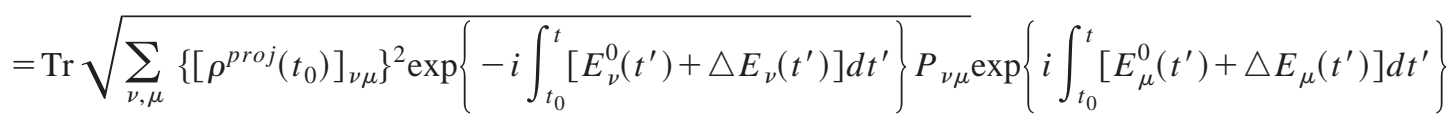

$$
\begin{aligned}
& =\sum_{\nu} \sqrt{\left\langle\phi_{\nu}\left|\sum_{\nu, \mu}\left\{\left[\rho^{p r o j}\left(t_{0}\right)\right]_{\nu \mu}\right\}^{2} \exp \left\{-i \int_{t_{0}}^{t}\left[E_{\nu}^{0}\left(t^{\prime}\right)+\Delta E_{\nu}\left(t^{\prime}\right)\right] d t^{\prime}\right\} P_{\nu \mu} \exp \left\{i \int_{t_{0}}^{t}\left[E_{\mu}^{0}\left(t^{\prime}\right)+\triangle E_{\mu}\left(t^{\prime}\right)\right] d t^{\prime}\right\}\right| \phi_{\nu}\right\rangle} \\
& =\sum_{\nu} \sqrt{\left\{\left[\rho^{\text {proj }}\left(t_{0}\right)\right]_{\nu \nu}\right\}^{2}}=\operatorname{Tr}\left(\sqrt{\rho^{p r o j}\left(t_{0}\right) \rho^{\text {proj }}\left(t_{0}\right)}\right)=1
\end{aligned}
$$

where $\left[\rho^{p r o j}\left(t_{0}\right)\right]_{\nu \mu}$ are the matrix elements of the density operator $\rho^{p r o j}\left(t_{0}\right)$. This exposes an exciting result: there is no decoherence in the projected subspace for states of the system since the eigenvectors remain invariant. However, a change in the eigenvalues introduces a phase shift in the evolution of the states,

$$
\exp \left[-i \int E_{\nu}^{0}(\tau) d \tau\right]\left|\psi_{\nu}^{p r o j}\left(t_{0}\right)\right\rangle \quad \text { After the interaction } \exp \left\{-i \int\left[E_{\nu}^{0}(\tau)+\Delta E_{\nu}(\tau)\right] d \tau\right\}\left|\psi_{\nu}^{p r o j}\left(t_{0}\right)\right\rangle .
$$

From these findings there are two conclusions,

(1) In general, for any system $S+B$, we can use the eigenprojectors of $H^{S}$ to construct a DF-projected subspace in which the eigenprojectors remain invariant before and after the interaction between $S$ and $B$, while the eigenvalues induce a phase shift in the eigenstates. The encoded states in the projected subspace are the projected states that are related to the original states by $P_{\nu} \Pi_{\nu} \psi_{\nu}$. In particular, in this projected subsystem, the states useful for performing quantum computing are the reduced projected states $\rho_{S}^{p r o j}(t)$. These can be obtained by using a projection operator $\operatorname{Tr}_{R} P_{\nu} \Pi_{\nu}$ in Liouville space to act on the density operator $\rho$ of the total system. Although $\rho_{S}^{p r o j}(t)$ is not a reduced density operator $\rho_{S}(t)$, one can consider $\rho_{S}^{p r o j}(t)$ to be a generalization of $\rho_{S}(t) \quad$ [i.e., when $\quad \Pi_{\nu} \rightarrow P_{\nu}, \quad \Sigma_{\nu} P_{\nu} \Pi_{\nu}$ $\left.\rightarrow 1, \rho_{S}^{\text {proj }}(t)=\operatorname{Tr}_{B} \Sigma_{\nu} P_{\nu} \Pi_{\nu} \rho(t)=\operatorname{Tr}_{B} \rho(t)=\rho_{S}(t)\right]$. We argue that the information encoded in this projected subspace can be measured because the projected bases are orthogonal and distinguishable in Liouville space, namely, $\left(\rho_{\nu}^{\text {proj,S }} \mid \rho_{\mu}^{\text {proj,S }}\right)$ $=0$, for $\nu \neq \mu$, because of $P_{\mu} P_{\nu}=0$ in Liouville space too.

(2) The phase shift induced by the eigenvalues may cost a unitary-type error for quantum computation. Although this sort of error may be eliminated by developing standard quantum-error correction schemes (such as multiqubit code [24]), the error recovery is not easy since phase error induced by the systematic phase shift may occur, at the same time, to many different clusters (inducing a phase or bit-flip errors in the encoded data). The increase in the number of phase errors for different qubits will cause a fast increase in the number of clusters, which likely leads to impractical implementations. On the other hand, the property of invariant eigenvectors in subspace provides the possibility of eliminating the unitary error by adjusting the appropriate time scale for the evolution operator to remain invariant under certain conditions, e.g., by choosing the time delay $\Delta t$ to allow $\Sigma_{\nu} \exp \left[-i \int_{0}^{t} E_{\nu}^{0}(\tau) d \tau\right] P_{\nu}=\Sigma_{\nu} \exp \left\{-i \int_{0}^{t+\Delta t}\left[E_{\nu}^{0}(\tau)\right.\right.$ $\left.\left.+\Delta E_{\nu}(\tau)\right] d \tau\right\} P_{\nu}$. Below, a concrete application of these concepts is presented.

\section{COMPUTING SYSTEM}

We consider a two-qubit quantum-computing system $S$, consisting of spins $\mathbf{S}_{1}$ and $\mathbf{S}_{2}$, such as those corresponding to two electrons around two ${ }^{31} P$ confined in a germanium/ silicon heterostructures in an electron spin-resonance transistor [4], or for two electrons confined in two quantum dots $[2,3]$. Ignoring the influence of the environment, the Hamiltonian may be written using the Heisenberg model as

$$
H_{S}(t)=J(t) \mathbf{S}_{1} \cdot \mathbf{S}_{2},
$$

where $J(t)$ is the time-dependent exchange coupling parameter determined by the specific model considerations. In the case of spins of the two electrons [e.g., confined in two vertically (laterally) coupled quantum dots], $J$ is the difference in the energies of two-electrons ground state, a spin singlet at zero magnetic field, and the lowest spin-triplet state, $J$ is also a function of the electric and magnetic field and the interdot distance $[25,26]$. Using the relationship between $\mathbf{S}_{1} \cdot \mathbf{S}_{2}$ and the square of the sum of $\mathbf{S}_{1}$ and $\mathbf{S}_{2}$, the eigenvalues and eigenvectors of $\mathbf{S}_{1} \cdot \mathbf{S}_{2}$ can be found from $\mathbf{S}_{1} \cdot \mathbf{S}_{2}=\frac{1}{2}\left(\mathbf{S}^{2}-\frac{3}{2}\right)$ by

$$
E_{S}^{1}=\frac{1}{4} \text { for }\left\{\begin{array}{c}
\left|\phi_{1}\right\rangle=|11\rangle, \\
\left|\phi_{2}\right\rangle=|00\rangle, \\
\left|\phi_{3}\right\rangle=\frac{1}{\sqrt{2}}(|01\rangle+|10\rangle),
\end{array}\right.
$$




$$
E_{S}^{2}=-\frac{3}{4} \text { for }\left|\phi_{4}\right\rangle=\frac{1}{\sqrt{2}}(|01\rangle-|10\rangle) \text {. }
$$

A quantum XOR gate is given by the sequence of operations [2], $U_{\mathrm{XOR}}=e^{i(\pi / 2) S_{1}^{z}} e^{-i(\pi / 2) S_{2}^{z}} U_{s w}^{1 / 2} e^{i \pi S_{1}^{z}} U_{s w}^{1 / 2}$, where $U_{s w}$ is an (ideal) swap operator and determined generally by an evolution operator $U_{s}(J(0) \tau)$ by adjusting the coupling time between the two spins in the evolution of the system. For the particular spin-spin coupling duration, $\tau_{s}$ where $\int_{0}^{\tau_{s}} J(t) d t$ $=\pi(\bmod 2 \pi), U_{s w}=U_{s}(\pi)$, the swap operator is given by

$$
\begin{aligned}
U_{s w}= & \exp \left[-i \int_{0}^{\tau_{s}} H_{S}(\tau) d \tau\right]=\sum_{j=1}^{3} e^{-i(\pi / 4)}\left|\phi_{j}\right\rangle\left\langle\phi_{j}\right| \\
& +e^{i(3 \pi / 4)}\left|\phi_{4}\right\rangle\left\langle\phi_{4}\right|
\end{aligned}
$$

and can exchange the quantum states of qubit 1 and 2 .

If we consider the influence of the environment, the nonideal action of the swap operation must be considered because the influence of environment introduces decoherence. To treat this decoherence, one needs to understand the behavior of the evolution of the system. Here it is assumed that the environment consists of a set of harmonic oscillators whose Hamiltonian is given by $H_{B}=\Sigma_{k} \omega_{k} b_{k}^{\dagger} b_{k}$, and the Hamiltonian coupling to the two-qubit spin system is $H_{i n t}$ $=\lambda \Sigma_{k}\left(\sigma_{z}^{1}+\sigma_{z}^{2}\right)\left(g_{k} b_{k}^{\dagger}+g_{k}^{*} b_{k}\right)$, where $b_{k}^{\dagger}, b_{k}$ are bosonic operators for the $k$ th field mode, characterized by a generally complex coupling parameter $g_{k}$, which characterizes the case as being one of either independent or collective decoherence [27]. The Hamiltonian operator for the total system is given by $H(t)=H_{S}(t)+H_{B}+\lambda H_{i n t}$, and the corresponding Schrödinger equation is $i \partial / \partial t \psi(t)=\left(H_{S}(t)+H_{B}\right.$ $\left.+H_{\text {int }}\right) \psi(t)$. Choosing the time-independent eigenprojectors of $H_{S}(t)+H_{B}$ as $P_{\nu}$ and $Q_{\nu}$ with $Q_{\nu}+P_{\nu}=1$, with

$$
P_{\nu} \equiv\left|\varphi_{\nu}\right\rangle\left\langle\varphi_{\nu}|=| n_{1} \cdots n_{k} \cdots\right\rangle\left|\phi_{j}\right\rangle\left\langle\phi_{j}\right|\left\langle\cdots n_{k} \cdots n_{1}\right|
$$

for $\nu=\left(j, n_{1} \cdots n_{k} \cdots\right)$ and $j=1, \ldots, 4 ; n_{k}=1, \ldots$, respectively, then the eigenprojectors $\Pi_{\nu}(t)$ for the total Hamiltonian $H(t)$ can be written in terms of the Heisenberg equation and satisfy the usual properties of projection operators.

\section{CODE CORRECTION IN SUBSPACE}

Using above subdynamics formulation, it is apparent that before the interaction occurs, the spectral decomposition for $\left[H_{S}(t)+H_{B}\right]$ is the same as that for the total intermediate operator $\Theta(t)$, i.e.,

$$
\begin{aligned}
H_{S}(t)+H_{B} & =\Theta(t) \\
& =\sum_{j=1}^{4} \sum_{n_{1} . . n_{k} \ldots} E_{j, n_{1} \cdots n_{k} \ldots}^{0} \ldots(t) P_{j, n_{1} \cdots n_{k} \ldots,},
\end{aligned}
$$

where $E_{j, n_{1} \cdots n_{k} \ldots}^{0}(t)=\left(\frac{1}{4}-\delta_{j 4} \frac{3}{4}\right) J(t)+\Sigma_{k} \omega_{k} n_{k}$. But once interaction with the environment occurs, the eigenvalues change from $E_{j, n_{1} \cdots n_{k} \ldots}^{0}(t)$ to $E_{j, n_{1} \cdots n_{k} \ldots}^{0}(t)$ $+\Delta E_{j, n_{1} \cdots n_{k}} \ldots(t)$, where we have made the following definitions:

$$
\begin{aligned}
\Delta E_{j, n_{1} \cdots n_{k}} \ldots(t)= & \left\langle n_{1} \cdots n_{k} \cdots\right|\left\langle\phi_{j} \mid H_{i n t} C_{j, n_{1} \cdots n_{k} \ldots(t)\left|\phi_{j}\right\rangle}\right\rangle \\
& \times\left|n_{1} \cdots n_{k} \cdots\right\rangle
\end{aligned}
$$

while the corresponding eigenvectors of $\Theta(t)$, $\left|\phi_{j}\right\rangle\left|n_{1} \cdots n_{k} \cdots\right\rangle$ remain invariant. The spectral decomposition of the intermediate operator $\Theta(t)$ is, thus, different from $H_{0}(t)$,

$$
\begin{aligned}
\Theta(t)= & \sum_{j=1}^{4} \sum_{n_{1}} \ldots n_{k} \ldots \\
& +\Delta E_{\left.j, n_{1} \cdots n_{k} \ldots(t)\right] P_{j, n_{1}} \cdots n_{k} \cdots} \cdots
\end{aligned}
$$

This shows that there is no decoherence in the projected subspace for the stationary states of the system since the eigenvectors remain invariant, but for the evolutionary states, the change of the eigenvalues can introduce a type of unitary-like error in the system evolution,

$$
\begin{aligned}
\left|\psi^{p r o j}(t)\right\rangle= & \sum_{j=1}^{4} \sum_{n_{1} \cdots n_{k} \ldots} \exp \left\{-i \int_{0}^{t}\left[E_{j, n_{1} \cdots n_{k} \ldots(\tau)}^{0}\right.\right. \\
& \left.\left.+\Delta E_{j, n_{1} \cdots n_{k} \ldots}(\tau)\right] d \tau\right\}\left|\psi_{j, n_{1} \cdots n_{k} \ldots}^{p r o j}(0)\right\rangle .
\end{aligned}
$$

As mentioned previously, the quantum XOR operator can be constructed from a sequence of operations related to the ideal swap operator, adjusted by controlling the coupling time for the interaction between the two-qubits without any influence from the environment. But if the effects of the environment are now included, the ideal swap operator changes to the nonideal swap operator, owing to the unitary error. To cancel this type of decoherence, in terms of the subdynamics formulation, it is proposed that one allow the quantum logical operators to work on the projected subspaces. For example, if the quantum XOR operator previously introduced is considered, the ideal swap operator should be adjusted by controlling the coupling time between the two spins without considering interactions with the environment; then the ideal swap operator is given in a projected subspace as $U_{s w}\left(\tau_{s}\right)=\exp \left[-i\left(\pi \mathbf{S}_{1} \cdot \mathbf{S}_{2} \otimes I_{B}+I_{S} \otimes H_{B} \tau_{s}\right)\right]$, where a specific coupling duration $\tau_{s}$ is given by $\int_{0}^{\tau_{s}} J(\tau) d \tau$ $=\pi(\bmod 2 \pi)$. The unitary error is related to the nonideal action of the swap operator which can be adjusted by the evolution operator in a projected subspace as $\quad U_{s w}^{\prime}\left(\tau_{s}+\Delta t\right)=\exp \left\{-i \int_{0}^{\tau_{s}+\Delta t} d \tau\left[J(\tau) \mathbf{S}_{1} \cdot \mathbf{S}_{2} \otimes I_{B}+I_{S} \otimes H_{B}\right.\right.$

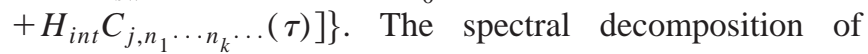
$U_{s w}^{\prime}\left(\tau_{s}+\Delta t\right)$ can be expressed by adjusting the interaction time $\tau_{s}$ to $\tau_{s}+\Delta t$, allowing the nonideal swap operator to be equal to the ideal swap operator, 


$$
\begin{aligned}
& U_{s w}^{\prime}\left(\tau_{s}+\Delta t\right)=U_{s w} \\
& =\sum_{n_{1}} \cdots n_{n_{k}} \ldots\left(\sum _ { j = 1 } ^ { 3 } \operatorname { e x p } \left\{-i \int_{0}^{\tau_{s}+\Delta t} d \tau\left[\frac{1}{4} J(\tau)+\sum_{k} \omega_{k} n_{k}+\Delta E_{\left.j, n_{1} \cdots n_{k} \cdots(t)\right]} P_{j, n_{1} \cdots n_{k} \cdots}\right.\right.\right. \\
& \left.+\exp \left\{-i \int_{0}^{\tau_{s}+\Delta t} d \tau\left[-\frac{3}{4} J(\tau)+\sum_{k} \omega_{k} n_{k}+\Delta E_{j, n_{1} \cdots n_{k}} \ldots(t)\right]\right\} P_{4, n_{1} \cdots n_{k} \cdots}\right\} \\
& =\sum_{n_{1} \cdots n_{k} \cdots}\left(\sum_{j=1}^{3} \exp \left(-i \frac{\pi}{4}-i \tau_{s} \sum_{k} \omega_{k} n_{k}\right) P_{j, n_{1} \cdots n_{k} \ldots}+\exp \left(i \frac{3 \pi}{4}-i \tau_{s} \sum_{k} \omega_{k} n_{k}\right) P_{\left.4, n_{1} \cdots n_{k} \cdots\right)} .\right.
\end{aligned}
$$

This induces the integral equation for determining $\Delta t$, which depends on $j, n_{1} \cdots n_{k} \cdots$,

$$
\begin{aligned}
& \int_{0}^{\tau_{s}+\Delta t} d \tau\left(\frac{J(\tau)}{4}-\delta_{j 4} \frac{3 J(\tau)}{4}+\Delta E_{\left.j, n_{1} \cdots n_{k} \ldots(\tau)\right)}\right. \\
& = \begin{cases}\frac{\pi}{4} \text { for } j=1,2,3, \\
-\frac{3 \pi}{4}, & \text { for } j=4 .\end{cases}
\end{aligned}
$$

For instance, if the exchange interaction coupling $J(\mathbf{B}, \mathbf{E}, d)$ is a time-independent function of the external magnetic field $\mathbf{B}$, electric field $\mathbf{E}$ and the interdot distance $d$ as in the case of vertically or laterally tunnel-coupled quantum dots, then $\Delta t$ can be solved from Eq. (22) as

$$
\Delta t=-\frac{\tau_{s}}{\frac{J(\mathbf{B}, \mathbf{E}, d)}{4 \Delta E_{j, n_{1} \cdots n_{k} \cdots} \cdots}+1}=-\frac{\tau_{s}}{\frac{-3 J(\mathbf{B}, \mathbf{E}, d)}{4 \Delta E_{4, n_{1} \cdots n_{k} \cdots} \cdots}, 1},
$$

where the concrete formula for $J(\mathbf{B}, \mathbf{E}, d)$, in the case of vertically or laterally tunnel-coupled quantum dots, can be found in Refs. [25,26]. Assuming, in this case, that the energy of the total system is uniformly distributed, i.e.,

$$
\begin{gathered}
\frac{J(\mathbf{B}, \mathbf{E}, d)}{4 \Delta E_{j, n_{1}} \cdots n_{k} \cdots}=\frac{-3 J(\mathbf{B}, \mathbf{E}, d)}{4 \Delta E_{4, n_{1} \cdots n_{k}} \cdots}=\mathrm{const} \\
\text { for } j=1,2,3 ; n_{1}, \ldots, n_{k}=1,2, \ldots
\end{gathered}
$$

The shift energy can be obtained from Eq. (18),

$$
\Delta E_{j, n_{1} \cdots n_{k} \cdots}=\sum_{j^{\prime}=1}^{4} \sum_{n_{1}^{\prime} \cdots n_{k}^{\prime} \cdots}\left(H_{i n t}\right)_{j, n_{1} \cdots n_{k} \cdots ; j^{\prime}, n_{1}^{\prime} \cdots n_{k}^{\prime} \cdots} C_{j^{\prime}, n_{1}^{\prime} \cdots n_{k}^{\prime} \cdots ; j, n_{1} \cdots n_{k} \cdots},
$$

and the matrix of the creation operator is given by

$$
C_{j^{\prime}, n_{1}^{\prime} \cdots n_{k}^{\prime} \cdots ; j, n_{1} \cdots n_{k} \cdots}=\left\langle\varphi_{j^{\prime}, n_{1}^{\prime} \cdots n_{k}^{\prime} \cdots}\left|C_{j, n_{1} \cdots n_{k} \cdots}\right| \varphi_{j, n_{1} \cdots n_{k} \cdots} \cdots,\right.
$$

which can be calculated from formula (6); for example, the first order $C_{j, n_{1} \cdots n_{k}}^{[1]}$. is divided by degenerate part and nondegenerate part,

$$
\begin{aligned}
& C_{j, n_{1} \cdots n_{k} \cdots}^{[1]}=\sum_{n_{1}^{\prime} \cdots n_{k}^{\prime} \cdots}\left[\sum_{j^{\prime} \neq j}^{3} \frac{1}{\Delta E_{j, n_{1} \cdots n_{k} \cdots}^{[2]}}\left(H_{i n t}\right)_{j^{\prime}, n_{1}^{\prime} \cdots n_{k}^{\prime} \cdots ; j, n_{1} \cdots n_{k} \cdots}\right. \\
& \left.+\delta_{j^{\prime} 4} \frac{1}{E_{j, n_{1} \cdots n_{k} \cdots}^{0}-E_{j^{\prime}, n_{1}^{\prime} \cdots n_{k}^{\prime} \cdots}^{0}}\left(H_{i n t}\right)_{j^{\prime}, n_{1}^{\prime} \cdots n_{k}^{\prime} \cdots ; j, n_{1} \cdots n_{k} \cdots}\right] P_{j^{\prime}, n_{1}^{\prime} \cdots n_{k}^{\prime} \cdots ; j, n_{1} \cdots n_{k} \cdots}
\end{aligned}
$$

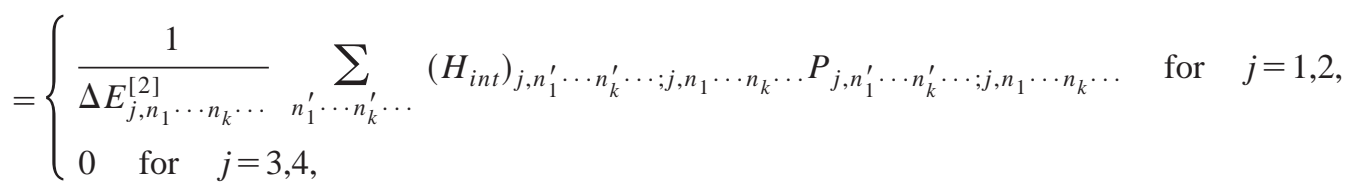

which results in the second order $\Delta E_{j, n_{1} \cdots n_{k} \ldots}^{[2]}$ by Eq. (25) 


$$
\Delta E_{j, n_{1} \cdots n_{k} \cdots}^{[2]}=\left\{\begin{array}{l}
\sqrt{\sum_{j=1}^{2} \sum_{n_{1}^{\prime} \cdots n_{k}^{\prime} \cdots}\left(\left(H_{i n t}\right)_{\left.j, n_{1}^{\prime} \cdots n_{k}^{\prime} \cdots ; j, n_{1} \cdots n_{k} \cdots\right)^{2}},\right.} \\
0 \quad \text { for } j=3,4
\end{array}\right.
$$

with the matrix elements given by

$$
\begin{aligned}
\left(H_{i n t}\right)_{j, n_{1} \cdots n_{k} \cdots ; j^{\prime}, n_{1}^{\prime} \cdots n_{k}^{\prime} \cdots} & =\left\langle n_{1} \cdots n_{k} \cdots\left|\left\langle\phi_{i}\left|H_{i n t}\right| \phi_{j^{\prime}}\right\rangle\right| n_{1}^{\prime} \cdots n_{k}^{\prime} \cdots\right\rangle \\
& =\left\{\begin{array}{l}
\lambda\left(\delta_{1, j^{\prime}}-\delta_{2, j^{\prime}}\right) \sum_{k}\left(\delta_{n_{k}, n_{k}^{\prime}+1} g_{k} \sqrt{n_{k}^{\prime}+1}+\delta_{n_{k}, n_{k}^{\prime}-1} g_{k}^{*} \sqrt{n_{k}^{\prime}}\right) \\
0 \quad \text { for } j=3,4 .
\end{array}\right.
\end{aligned}
$$

Equation (23) shows that although the interaction introduces the sort of phase shift in the swap operator, this sort of phase shift can be canceled by adjusting the coupling time between the two spins under the assumptions of homogeneous distribution of energy (i.e., owing to the invariance of the eigenvectors in the projected subspace). In the same way, the second-order projected states in the projected subspace are given by

$$
\begin{aligned}
& \psi_{j, n_{1} \cdots n_{k} \ldots}^{p r o j[2]}(t)=\left(P_{j, n_{1} \cdots n_{k} \ldots}+D_{j, n_{1} \cdots n_{k} \cdots}-D_{j, n_{1} \cdots n_{k} \ldots} C_{j, n_{1} \cdots n_{k} \cdots}\right)|\psi(t)\rangle
\end{aligned}
$$

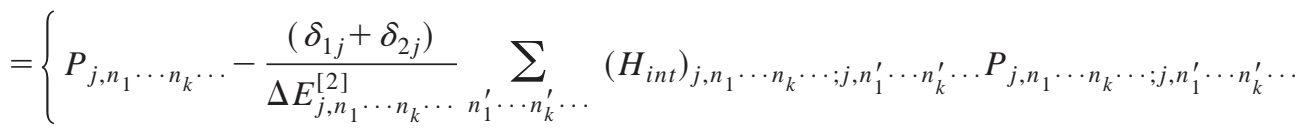

$$
\begin{aligned}
& -\frac{\left(\delta_{1 j}+\delta_{2 j}\right)}{\left(\Delta E_{j, n_{1} \cdots n_{k} \cdots}^{[2]}\right)^{2}} \sum_{n_{1}^{\prime} \cdots n_{k}^{\prime} \cdots n_{1}^{\prime \prime} \cdots n_{k}^{\prime \prime} \cdots}\left[\left(H_{i n t}\right)_{j, n_{1} \cdots n_{k} \cdots ; j, n_{1}^{\prime} \cdots n_{k}^{\prime} \cdots}\right.
\end{aligned}
$$

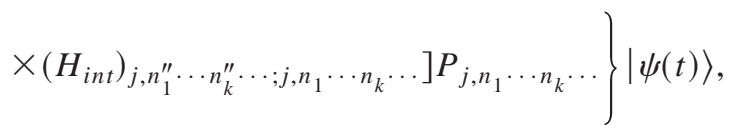

where the first-order destruction operator is

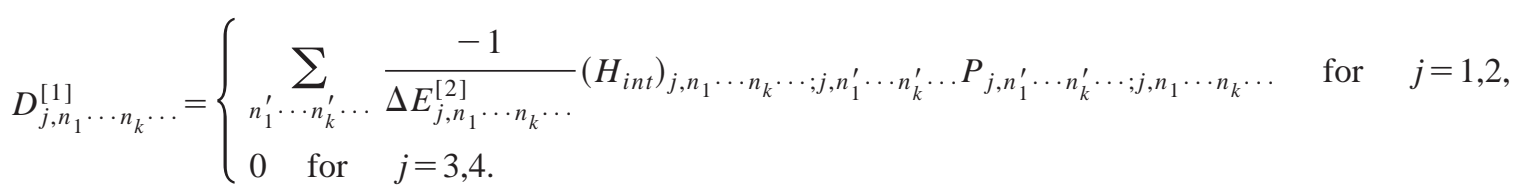

By the above canceling procedure, the evolution formula for the second-order reduced projected density operator, before or after the interaction, remains the same and is given by

$$
\begin{aligned}
\rho_{j, n_{1} \cdots n_{k} \cdots ; j^{\prime}, n_{1} \cdots n_{k} \cdots}^{\operatorname{proj}[2], S}(t)= & \operatorname{Tr}_{B}\left\{\operatorname { e x p } \left(-i \Theta_{j, n_{1} \cdots n_{k} \cdots t}^{[2]} p_{j, n_{1} \cdots n_{k} \cdots ; j^{\prime}, n_{1}^{\prime} \cdots n_{k}^{\prime} \cdots}\right.\right. \\
& \left.\times\left|\varphi_{j, n_{1} \cdots n_{k} \cdots}^{\operatorname{proj}[2]}(0)\right\rangle\left\langle\varphi_{j^{\prime}, n_{1}^{\prime} \cdots n_{k}^{\prime} \cdots}^{\operatorname{proj}[2]}(0)\right| \exp \left(i \Theta_{j^{\prime}, n_{1}^{\prime} \cdots n_{k}^{\prime} \cdots}^{[2]} t\right)\right\} \\
= & \exp \left[-i\left(E_{j}^{0}-E_{j^{\prime}}^{0}\right) t\right] \rho_{j, n_{1} \cdots n_{k} \cdots ; j^{\prime}, n_{1} \cdots n_{k} \cdots}^{p r o j, S}(0),
\end{aligned}
$$

where $p_{j, n_{1} \cdots n_{k} \cdots ; j^{\prime}, n_{1}^{\prime} \cdots n_{k}^{\prime} \cdots}$ represent probability of existence for the state $\left|\varphi_{j, n_{1} \cdots n_{k} \cdots}^{\operatorname{proj}[2]}(0)\right\rangle\left\langle\varphi_{j^{\prime}, n_{1}^{\prime} \cdots n_{k}^{\prime} \cdots(0) \mid \text {, noting that }}^{\operatorname{roj}[2]}\right.$

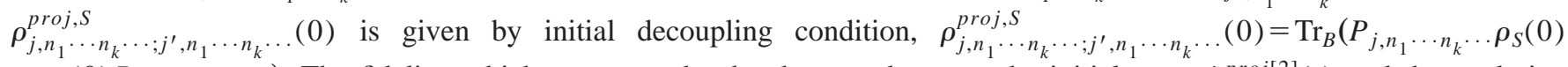

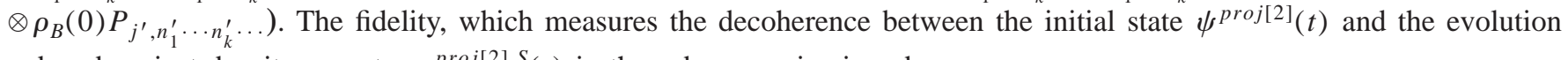
reduced project density operator $\rho^{\operatorname{proj[2],S}}(t)$ in the subspaces, is given by 


$$
\begin{aligned}
& F\left[\left|\psi^{\operatorname{proj[2]}}(t)\right\rangle, \rho^{\operatorname{proj}[2], S}(t)\right] \\
& =\sqrt{\left\langle\psi^{p r o j[2]}(t)\left|\sum_{j, j^{\prime}, n_{1} \cdots n_{k} \cdots} \rho_{j, n_{1} \cdots n_{k} \cdots ; j^{\prime}, n_{1} \cdots n_{k} \cdots}^{\text {proj[2],S }}(t)\right| \psi^{p r o j[2]}(t)\right\rangle} \\
& =\sqrt{\left.\sum_{j, n_{1} \cdots n_{k} \cdots} \varphi_{j, n_{1} \cdots n_{k} \cdots}^{p r o j[2]}(0)\left|\sum_{j, j^{\prime}, n_{1} \cdots n_{k} \cdots} \rho_{j, n_{1} \cdots n_{k} \cdots ; j^{\prime}, n_{1} \cdots n_{k} \cdots}^{p r o j[2]}(0)\right| \sum_{j^{\prime}, n_{1} \cdots n_{k} \cdots} \varphi_{j^{\prime}, n_{1}^{\prime} \cdots n_{k}^{\prime} \cdots}^{\operatorname{proj}[2]}(0)\right\rangle} \\
& =\sqrt{\sum_{j, n_{1} \cdots n_{k} \cdots} p_{j, n_{1} \cdots n_{k} \cdots \sum_{j^{\prime}, n_{1} \cdots n_{k} \cdots} \sum_{j^{\prime}, n_{1} \cdots n_{k} \cdots}}}=1 \text {, }
\end{aligned}
$$

which shows that there is no decoherence after the cancellation process.

\section{THE DF PROJECTED SUBSPACE}

A completely decoherence-free condition (i.e., including no phase shift) in the projected subspace can also be determined, due to the definition of the intermediate operator of Eq. (2). That is,

$$
H_{\text {int }} C(t)=0 .
$$

In this case, eigenvalues for the intermediate operators in the projected subspaces, are the same as those for the total Hamiltonian in the total space, while the eigenvectors are the same as those of the unperturbed Hamiltonian. The projected subspaces spanned by the set of the eigenvectors of the intermediate operator are invariant for the evolutionary and stationary states of the system, and are closed for the intermediate operators. This allows quantum computing with co- herent states such that no information leaks out from the projected subspaces. Hence one can use the full power of DF subspaces to preserve coherence for full-scale quantum computing. The key question then is how to ensure that Eq. (34) holds. In the Born-Markov approximation (e.g., Lindbladequation approach) and the assumptions of symmetric or collective decoherence $[6,7,13,24]$, if $P_{\mu}$ are partial eigenprojectors of $H_{\text {int }}$ (i.e., $H_{\text {int }} P_{\mu}=\varepsilon_{\alpha} P_{\mu}, \forall$ degenerate $\alpha \in$ set of complex numbers $c$ ) then it means having $P_{\nu} H_{\text {int }} P_{\mu} C_{\nu}(t) P_{\nu}=0$, for any $\nu$. But in the general case, without restrictions on the type of decoherence (e.g., a nonMarkovian process and nonsymmetric and noncollective decoherence) how can one find the conditions for constructing the DF subspace? For generality, we propose a procedure to find the condition for the DF subspace starting directly from Eq. (34) by considering weak coupling between the system and the environment, without making any of the above assumptions. In fact, from Eq. (34) we have

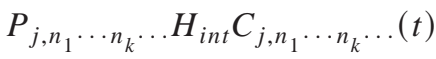

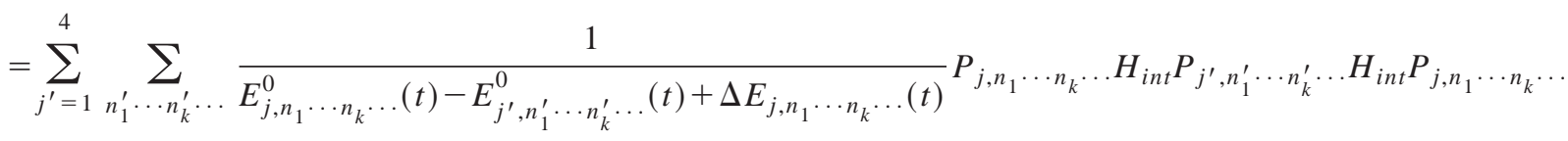

$$
\begin{aligned}
& =\frac{\lambda^{2}}{E_{1, n_{1} \cdots n_{k} \cdots}^{0} \ldots(t)-E_{1, n_{1} \cdots n_{k}^{\prime} \cdots}^{0}(t)+\Delta E_{1, n_{1} \cdots n_{k} \cdots(t)}} \sum_{k}\left[g_{k} g_{k+1}^{*}\left(n_{k}+1\right)+g_{k-1} g_{k}^{*} n_{k}\right] \delta_{j 1} P_{1, n_{1} \cdots n_{k} \cdots} \\
& +\frac{\lambda^{2}}{E_{2, n_{1} \cdots n_{k} \ldots}^{0} \ldots(t)-E_{2, n_{1} \cdots n_{k}^{\prime} \cdots}^{0}(t)+\Delta E_{2, n_{1} \cdots n_{k} \ldots(t)}} \sum_{k}\left[g_{k} g_{k+1}^{*}\left(n_{k}+1\right)+g_{k-1} g_{k}^{*} n_{k}\right] \delta_{2 j} P_{2, n_{1} \cdots n_{k} \ldots}=0 .
\end{aligned}
$$

This gives a DF condition, which is a restriction on the bath operator by

$$
\sum_{k}\left[g_{k} g_{k+1}^{*}\left(n_{k}+1\right)+g_{k-1} g_{k}^{*} n_{k}\right]=0 .
$$

In one spectial case it is

$$
\frac{n_{k}+1}{n_{k}}=-\frac{g_{k-1} g_{k}^{*}}{g_{k} g_{k+1}^{*}}
$$

Under the DF conditions, e.g., with Eq. (36) or (37) holding, Eq. (1) reduces to 


$$
i \frac{\partial\left|\varphi^{p r o j}(t)\right\rangle}{\partial t}=H_{0}(t)\left|\varphi^{p r o j}(t)\right\rangle
$$

with evolution of the projected state described by

$$
\begin{aligned}
\left|\varphi^{p r o j}(t)\right\rangle= & \hat{T} e^{-i t H_{0}(t)}\left|\varphi^{p r o j}(0)\right\rangle \\
= & \hat{T} e^{-i t H_{0}(t)} \sum_{\nu}\left[P_{\nu}+D_{\nu}(0)\right]^{-1} \\
& \times\left[P_{\nu}+D_{\nu}(0)\right]|\varphi(0)\rangle,
\end{aligned}
$$

which enables the constructed projected subspaces to become a DF subspace spanned by the set of $\left\{P_{j, n_{1} \cdots n_{k} \cdots}\right\}$, although the total space is subject to decoherence. The projected subspace is closed with respect to the intermediate operator, $\Theta_{v}(t) \Phi^{p r o j} \subset \Phi^{p r o j}$, for any projected state in the projected subspace $\Phi^{p r o j},\left(P_{\nu} \Pi_{\nu}(t) \psi(t)\right)$. Furthermore, if one generalizes the initial-test projector $P_{\mu}$ to be up (or down) triangular from the partial eigenprojector of $H_{\text {int }}$, i.e., $P_{\nu} H_{\text {int }} P_{\mu} \neq 0$ for $\nu<\mu$, otherwise it is zero, then Eq. (2) gives $C_{\nu}(t) P_{\nu}=0$, which enables Eq. (34) to generally hold in the projected subspace without any approximation. In this sense, if one can construct a triangular basis for $H_{\text {int }}$ to span a projected subspace, then this space is DF although the total space is decoherent.

\section{REALIZATION OF A DF SUBSPACE USING TRIANGULATION}

As a starting point we consider a necessary and sufficient condition for DF behavior in the projected subspace. This can be determined from the fact that the interaction part of the intermediate operator $\Theta(t)$ is zero or that the evolution of the projected (reduced) state is independent of the interaction part of the Liouvillian. That is,

$$
L_{0}^{i n t}+\left[L_{1}^{S}(t)+L_{1}^{B}+L_{1}^{i n t}\right] C(t)=0 .
$$

If the constructed projectors $P_{\nu}$ and $Q_{\nu}$ are triangular with respect to $L_{1}^{S}(t)+L_{1}^{B}+L_{1}^{\text {int }} \quad$ [i.e., $\quad P_{\nu} L_{\text {int }} P_{\mu}$ $\neq 0, P_{\nu}\left(L_{1}^{S}(t)+L_{1}^{B}\right) P_{\mu} \neq 0$ for $\nu<\mu$ (up triangular) or $\nu>\mu$ (down triangular) $]$, then $L_{0}^{\text {int }}+\left[L_{1}^{S}(t)+L_{1}^{B}+L_{1}^{\text {int }}\right] C(t)$ $=L_{0}^{i n t}+\Sigma_{\nu} P_{\nu}\left(L_{1}^{S}(t)+L_{1}^{B}+L_{1}^{i n t}\right) Q_{\nu} 1 /\left(E_{\nu}(t)-Q_{\nu} L(t) Q_{\nu}\right) Q_{\nu}$ $\left(L_{1}^{S}(t)+L_{1}^{B}+L_{1}^{i n t}\right) P_{\nu}=0$. This enables the evolution of the reduced projected density operator to be independent of the interaction part of the total Liouvillian in the projected Liouville subspace,

$$
\begin{aligned}
\rho^{p r o j, S}(t) & =\sum_{\nu} \operatorname{Tr}_{B}\left[\left\{P_{\nu}+D_{\nu}(t)\right\} \rho(t)\right] \\
& =\sum_{\nu} \operatorname{Tr}_{B}\left\{\exp \left[-i \int_{t_{0}}^{t} d t^{\prime} l_{\nu}^{\Theta_{0}}\left(t^{\prime}\right)\right] P_{\nu} \rho\left(t_{0}\right)\right\},
\end{aligned}
$$

where $l_{\nu}^{\Theta_{0}}(t)$ is a $\nu$ th eigenvalue of $\Theta_{0}(t)$ in Liouvillian representation. The corresponding mixed-state fidelity is equal to 1 \{i.e., $F(t)=\operatorname{Tr}_{S}\left[\rho^{p r o j, S}(0) \Sigma_{\nu} \operatorname{Tr}_{B}(\exp \right.$ $\left.\left.\left.\left[-i \int_{t_{0}}^{t} d t^{\prime} l_{\nu}^{\Theta_{0}}\left(t^{\prime}\right)\right] P_{\nu} \rho\left(t_{0}\right)\right)\right]=1\right\}$. This shows that there is no de- coherence introduced by the environment in the projected subspace, although the total system is subject to decoherence introduced by the environment. The formulation for this DFprojected subspace is exact (i.e., there are no approximations including the Born-Markov approximation). The method is general. Thus, for any combined system, one may construct a triangular basis for the total Hamiltonian to span a projected subspace in which the evolution of the projected density operator is independent of the interaction part of the total Hamiltonian, with a fidelity of 1 . Furthermore, one can construct a partial triangular basis for the computing system or bath, by choosing a partial diagonal basis for the left part of total system to form a DF-projected subspace, based on the subdynamical Liouville equation. Thus, triangulation procedure places no restriction on the type of coupling between the system and the environment (i.e., Markovian or collective decoherence).

\section{EXAMPLE}

To illustrate the above method, we revisit the example of the two-qubit quantum-computing system $S$, consisting of spins $\mathbf{S}_{1}$ and $\mathbf{S}_{2}$. For example, this includes the two electrons around two ${ }^{31} \mathrm{P}$ confined in a germanium/silicon heterostructures in an electron spin-resonance transistor [1], or two electrons confined in two quantum dots [2]. Ignoring the influence of environment, the Hamiltonian can be written using the Heisenberg model as $H_{S}(t)=J(t) \mathbf{S}_{1} \cdot \mathbf{S}_{2}$, where $J(t)$ is the time-dependent exchange coupling parameter determined by the specific model considerations. The coupling to the environment is assumed to be described by a CaldeiraLeggett-type model, consisting of a set of harmonic oscillators coupled linearly to $S$ by $H_{i n t}=\lambda \Sigma_{k=1}^{2} \boldsymbol{\sigma}_{k} \cdot \mathbf{b}_{k}$, where $b_{k}^{j}$ $=\Sigma_{\alpha} g_{\alpha}^{k j}\left(a_{\alpha, k j}+a_{\alpha, k j}^{\dagger}\right)$ is a fluctuation quantum field. The Hamiltonian of bath is given by $H_{B}=\Sigma_{\alpha} \omega_{\alpha}^{k j} a_{\alpha, k j}^{\dagger} a_{\alpha, k j}$, where $a_{\alpha, k j}^{\dagger}\left(a_{\alpha, k j}\right)$ are bosonic creation (annihilation) operators with $j=x, y, z$ and $\omega_{\alpha}^{k j}$ are the corresponding frequencies with spectral-distribution function $J_{k j}(\omega)=\pi \Sigma_{\alpha}\left(g_{\alpha}^{k j}\right)^{2} \delta(\omega$ $\left.-\omega_{\alpha}\right)[28]$.

We show how to triangulate the partial basis of the bath since, in principle, triangulating the partial basis of the system follows the same approach. The key point is that, in general, the triangular property of $L$ results in $P_{\nu} L Q_{\nu} C_{\nu} P_{\nu}$ being in the SKE.

The matrix for $H_{B}+H_{\text {int }}$, with respect to a $\alpha$ th element of the basis of $B$, in a repeat subspace, $\left(\left|n_{\alpha, k}^{j}\right\rangle \mid n_{\alpha, k}^{j}\right.$ $\left.+1\rangle\left\langle n_{\alpha, k}^{j}\right|\left\langle n_{\alpha, k}^{j}+1\right|\right)$, can be written as

$$
M \equiv\left(\begin{array}{cc}
\omega_{\alpha}^{k j} n_{\alpha, k}^{j} & g_{\alpha}^{k j} \sqrt{n_{\alpha, k}^{j}+1} \\
g_{\alpha}^{k j} \sqrt{n_{\alpha, k}^{j}+1} & \omega_{\alpha}^{k j}\left(n_{\alpha, k}^{j}+1\right)
\end{array}\right) .
$$

Using a similarity transformation to up triangulate $M$, 
$M^{\text {tri }}$

$$
\begin{aligned}
= & \frac{1}{a d-b c}\left(\begin{array}{cc}
d & -b \\
-c & a
\end{array}\right)\left(\begin{array}{cc}
\omega_{\alpha}^{k j} n_{\alpha, k}^{j} & g_{\alpha}^{k j} \sqrt{n_{\alpha, k}^{j}+1} \\
g_{\alpha}^{k j} \sqrt{n_{\alpha, k}^{j}+1} & \omega_{\alpha}^{k j}\left(n_{\alpha, k}^{j}+1\right)
\end{array}\right) \\
& \times\left(\begin{array}{cc}
a & b \\
c & d
\end{array}\right)=\frac{1}{a d-b c} \\
& \times\left(\begin{array}{cc}
a d \omega_{\alpha}^{k j} n_{\alpha, k}^{j} & \left(d^{2}-b^{2}\right) g_{\alpha}^{k j} \sqrt{n_{\alpha, k}^{j}+1}-b d \omega_{\alpha}^{k j} \\
0 & -b c \omega_{\alpha}^{k j}\left(n_{\alpha, k}^{j}+2\right)+a d \omega_{\alpha}^{k j}\left(n_{\alpha, k}^{j}+1\right)
\end{array}\right),
\end{aligned}
$$

we have

$$
-b c \omega_{\alpha}^{k j}\left(n_{\alpha, k}^{j}+1\right)+(d c-a b) g_{\alpha}^{k j} \sqrt{n_{\alpha, k}^{j}+1}=0,
$$

$$
\left(-c^{2}+a^{2}\right) g_{\alpha}^{k j} \sqrt{n_{\alpha, k}^{j}+1}+a c \omega_{\alpha}^{k j}=0,
$$

which gives

$$
\begin{aligned}
& a=\left(\frac{-\omega_{\alpha}^{k j}}{2 g_{\alpha}^{k j} \sqrt{n_{\alpha, k}^{j}+1}} \pm \sqrt{\left(\frac{\omega_{\alpha}^{k j}}{2 g_{\alpha}^{k j}}\right)^{2} \frac{1}{n_{\alpha, k}^{j}+1}+1}\right) c \equiv \gamma_{\alpha, k j} c, \\
& d=\frac{\omega_{\alpha}^{k j} \sqrt{n_{\alpha, k}^{j}+1}+\gamma_{\alpha, k j} g_{\alpha}^{k j}}{g_{\alpha}^{k j}} b \equiv \zeta_{\alpha, k j} b .
\end{aligned}
$$

This determines the triangular basis for $H_{B}+H_{\text {int }}$ in the repeat subspace as $\left(\left|\phi_{n} \otimes\left\{n_{\alpha, k}^{j,{ }^{ \pm}}\right\}\right\rangle,\left\langle\left\{n_{\alpha, k}^{j,{ }^{ \pm}}\right\} \otimes \phi_{n}\right|\right)$. For example, by choosing $c=1$ and $b=-1$ we have

$$
M^{t r i}=\left(\begin{array}{cc}
-\gamma_{\alpha, k j} \zeta_{\alpha, k j} \omega_{\alpha}^{k j} n_{\alpha, k}^{j} & \left(\zeta_{\alpha, k j}^{2}-1\right) g_{\alpha}^{k j} \sqrt{n_{\alpha, k}^{j}+1}-\zeta_{\alpha, k j} \omega_{\alpha}^{k j} \\
0 & \omega_{\alpha}^{k j}\left(n_{\alpha, k}^{j}+2\right)-\gamma_{\alpha, k j} \zeta_{\alpha, k j} \omega_{\alpha}^{k j}\left(n_{\alpha, k}^{j}+1\right)
\end{array}\right),
$$

defining,

$$
\left(\begin{array}{c}
\left|n_{\alpha, k}^{j,+}\right\rangle \\
\left|n_{\alpha, k}^{j,-}\right\rangle
\end{array}\right)=\left(\begin{array}{c}
\gamma_{\alpha, k j}\left|n_{\alpha, k}^{j}\right\rangle-\left|n_{\alpha, k}^{j}+1\right\rangle \\
\left|n_{\alpha, k}^{j}\right\rangle-\zeta_{\alpha, k j}\left|n_{\alpha, k}^{j}+1\right\rangle
\end{array}\right)
$$

and

$$
\left(\begin{array}{c}
\left\langle n_{\alpha, k}^{j,+}\right| \\
\left\langle n_{\alpha, k}^{j,-}\right|
\end{array}\right)=\frac{1}{-\gamma_{\alpha, k j} \zeta_{\alpha, k j}+1}\left(\begin{array}{c}
-\zeta_{\alpha, k j}\left\langle n_{\alpha, k}^{j}\right|-\left\langle n_{\alpha, k}^{j}+1\right| \\
\left\langle n_{\alpha, k}^{j}\right|+\gamma_{\alpha, k j}\left\langle n_{\alpha, k}^{j}+1\right|
\end{array}\right)
$$

with $\left\{n_{\alpha, k}^{j, \pm}\right\}=\left(n_{1, k}^{j, \pm} \cdots n_{\alpha, k}^{j, \pm} \cdots\right)$. Then we can construct a triangular basis for the total Liouvillian $L(t)$ by $\left\{\| \phi_{n}\right.$ $\left.\left.\otimes\left\{n_{\alpha, k}^{j, \frac{ \pm}{ \pm}}\right\}\right\rangle\left\langle\left\{n_{\beta, k}^{j,{ }_{k}}\right\} \otimes \phi_{m}\right|\right),\left(\left|\phi_{m} \otimes\left\{n_{\beta, k}^{j,{ }^{*}}\right\}\right\rangle\left\langle\left\{n_{\alpha, k}^{j, \frac{ \pm}{B}}\right\} \otimes \phi_{n}||\right\}\right.$. This gives triangulation of matrix of $L_{B}+L_{i n t}$. By means of the new triangular basis one can define the projectors as $P_{\nu}$ $\left.\left.\equiv \| \phi_{n} \otimes\left\{n_{\alpha, k}^{j, \pm}\right\}\right\rangle\left\langle\left\{n_{\beta, k}^{j, \mp}\right\} \otimes \phi_{m}\right|\right)\left(\left|\phi_{m} \otimes\left\{n_{\beta, k}^{j, \overline{+}}\right\}\right\rangle\left\langle\left\{n_{\alpha, k}^{j,{ }^{ \pm}}\right\} \otimes \phi_{n} \|\right.\right.$ and $Q_{\nu} \equiv 1-P_{\nu}$. Taking into account the definitions of the creation and destruction operators, one has $P_{\nu}\left(L_{1}^{B}\right.$ $\left.+L_{1}^{i n t}\right) P_{\mu} C_{\nu}(t) P_{\nu} \sim P_{\nu}\left(L_{1}^{B}+L_{1}^{i n t}\right) P_{\mu}\left(L_{1}^{B}+L_{1}^{i n t}\right) P_{\nu} \rightarrow 0$ for $\mu>\nu$ (up-triangular property). Therefore, one finds the DF condition: $L_{0}^{i n t}+\left(L_{1}^{B}+L_{1}^{i n t}\right) C(t)=0$ with $\Theta(t)=\Theta_{0}(t)=L_{0}^{S}$ $+L_{0}^{B}$. Under this DF condition, the SKE reduces to

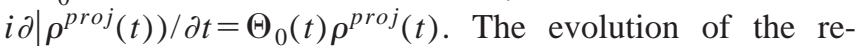
duced projected state, in the initial decoupling condition, becomes to Eq. (41). This shows that $\rho_{S}^{p r o j}(t)$ is independent on $L_{i n t}$. Therefore, there is no decoherence introduced by $L_{\text {int }}$ in the projected subsystem although the total system is subject to the decoherence introduced by $L_{i n t}$. In this projected subsystem, the quantum controlled-NOT logic operation is still given by a sequence of operations and the swap operator $U_{s w}$ remains invariant before and after the interaction, and is given by

$$
\begin{aligned}
\operatorname{Tr}_{B} \hat{T} \exp \left[-i \int_{0}^{\tau_{s}} \Theta(\tau) d \tau\right] \rho^{p r o j}(0) \\
=\operatorname{Tr}_{B} \hat{T} \exp \left[-i \int_{0}^{\tau_{s}} L_{0}^{S}(\tau) d \tau\right] \rho^{p r o j}(0) \\
=\operatorname{Tr}_{B}\left\{\hat{T} \exp \left[-i \int_{0}^{\tau_{s}} H_{0}^{S}(\tau) d \tau\right] \rho^{p r o j}(0)\right. \\
\\
\left.\quad \times \hat{T} \exp \left[i \int_{0}^{\tau_{s}} H_{0}^{S}(\tau) d \tau\right]\right\} \\
=\operatorname{Tr}_{B}\left[U_{s w} \rho^{p r o j}(0) U_{s w}^{-1}\right],
\end{aligned}
$$

where

$$
\begin{aligned}
U_{s w}= & \sum_{\alpha}\left(\sum_{n=1}^{3} e^{-i(\pi / 4)}\left|\phi_{n} \otimes \mathbf{1}_{\alpha}\right\rangle\left\langle\mathbf{1}_{\alpha} \otimes \phi_{n}\right|\right. \\
& \left.+e^{i(3 \pi / 4)}\left|\phi_{4} \otimes \mathbf{1}_{\alpha}\right\rangle\left\langle\mathbf{1}_{\alpha} \otimes \phi_{4}\right|\right) .
\end{aligned}
$$

The following table clarifies the difference between the total space and the projected subspace constructed by the following triangulation.
Initial state

Evolutionary state

$\operatorname{Tr}_{B} \hat{T} \exp \int L_{0}^{S}(t) d t P_{\nu} \rho_{S}(0) \otimes \rho_{B}(0)$

$\operatorname{Tr}_{B} T \exp \left\{-i \int\left(L_{0}^{S}(t)+L_{1}^{i n t}(t)\right] d t\right\} \rho_{S}(0) \otimes \rho_{B}(0)$
Fidelity

Subspace

$\operatorname{Tr}_{B} P_{\nu} \rho_{S}(0) \otimes \rho_{B}(0)$

Total space

$$
\operatorname{Tr}_{B} \rho_{S}(0) \otimes \rho_{B}(0)
$$

$f=1$

$0<f<1$ 
If one assumes that in the Schrödinger picture, an initial state of $S$ is $\phi_{S}(0)=\frac{1}{2}(|\uparrow \downarrow\rangle+|\downarrow \uparrow\rangle)-\frac{1}{2}(|\uparrow \uparrow\rangle+|\downarrow \downarrow\rangle)$, then by choosing a projector $P_{\nu} \quad$ as $\quad\left|\frac{1}{2}(|\uparrow \downarrow\rangle+|\downarrow \uparrow\rangle)\right.$ $\left.\otimes\left\{n_{\alpha, k}^{j, \pm}\right\}\right)\left(\left\{n_{\alpha, k}^{j, \pm}\right\} \otimes \frac{1}{2}(|\uparrow \downarrow\rangle+|\downarrow \uparrow\rangle) \mid\right.$, after taking into account the obtained triangular basis, an initial state in the DF $P_{\nu}$-projected subspace is given by $\operatorname{Tr}_{B} P_{\nu} \phi_{S}(0)=\frac{1}{2}(|\uparrow \downarrow\rangle$ $+|\downarrow \uparrow\rangle)$ under the initial decoupling condition. This correspondence is realized by the projection, $\phi_{S}(0) \rightarrow P_{\nu}^{S} \phi_{S}(0)$. The advantage of using $P_{\nu}^{S} \phi_{S}(0)$ compared with $\phi_{S}(0)$, is that the evolution of $P_{\nu}^{S} \phi_{S}(0)$ is independent of the interaction between $S$ and $B$ in the projected subspace. Therefore, one can encode information onto $P_{\nu}^{S} \phi_{S}(0)$ to perform quantum-computing (or quantum communication), protecting against decoherence introduced by the interaction between $S$ and $B$.

Finally, it should be noted that it is not necessary that the evolution operator $U_{\Theta}$ in the projected subspace is unitary, because a quantum-computing system projected in a DF subspace may be an open quantum system, obeying the semigroup-evolution rules [17]. In this open system, selfadjoint operators and unitary-evolution groups are not intrinsically necessary to govern quantum computation. Quantum computation can then be performed in a more general functional space, such as RHS, rather than just Hilbert space. The projected state $\psi^{p r o j}$ may exist in the test space $\Phi^{p r o j}$, which is a dense subspace of the Hilbert space $\mathcal{H}^{\text {proj }}$ constructed by $\Sigma_{\nu} P_{\nu} \Pi_{\nu} \mathcal{H}$, representing the physical states that can be prepared in an actual experiment. Its adjoint $\widetilde{\psi}^{p r o j}$ lies in the dual space $\left(\Phi^{p r o j}\right)^{\times}$, representing a procedure that associates with each state a number, while preserving the linear structure which results from the superposition principle, i.e., the triplet structure $\Phi^{p r o j} \subset \mathcal{H}^{p^{r o j}} \subset\left(\Phi^{p r o j}\right)^{\times}$[23]. This is a RHS structure that facilitates describing irreversible processes such as decoherence and dissipation due to interaction with the environment. In this space the evolution of the states are permitted to be time asymmetric, providing a framework for describing the irreversibility of practical open systems. This irreversibility does not change quantum-reversible logical operations to quantum-irreversible logical operation in the quantum universal Controlled-NOT logical gate. To appreciate this one must distinguish between irreversibility of a quantum logical operation, introduced by the structure of logical gate, and irreversibility of the process induced by interactions with the environment. Reversible computation means reversible logical operations on the structure of the logical gate. In this sense, quantum computing in RHS is compatible with reversible quantum logical operations and permits computing any reversible function, although irreversible processes do in fact exist.

\section{CONCLUSION}

In conclusion, a subdynamics-based formulation in the Schrödinger picture was presented for an open quantum system. Based on the subdynamical kinetic equation for an open quantum system, a proposal for quantum computing in the projected subspaces is developed. The eigenvectors of the intermediate operator in this subspace were shown to remain invariant before and after interaction with the environment, while the eigenvalues of the intermediate operator in this subspace change. The fidelity of mixed states in the projected subspace is 1 , which means that the constructed projected subspace is definitely DF. This reveals a universal property for any system plus reservoir: one can construct a DFprojected subspace by using eigenprojectors of the free part of the Hamiltonian in which the encoded states are projected states, which are themselves determined by relevant formulas of subdynamics. On the other hand, changes of the eigenvalues after interaction may introduce a type of unitary error in the ideal swap operator. This sort of error can be canceled by adjusting the interaction coupling time between two spins in the subspace, since the eigenvectors remain invariant, although decoherence exists in the total space for the total system. Finally, the general case for completely DF behavior in the projected subspaces was discussed, and it was shown that using a general DF condition (i.e., the second term of the subdynamics kinetic equation is zero), one can find a condition to allow the constructed projected subspace to be DF. This reveals that this condition is a necessary and sufficient condition for constructing a DF-projected subspace. We wish to emphasize two points here: (1) the constructed projected subspace is spanned by a set of $\left\{\rho_{v}^{p r o j}(t)\right\}$, which is closed with respect to the intermediate operator. Indeed, $\quad \Theta(t) \rho^{p r o j}(t)=\sum_{\nu} P_{\nu} L(t)\left(P_{\nu}+C_{\nu}(t)\right) \Pi_{\nu}(t) \rho(t)$ $=\Sigma_{\nu} l_{\nu}^{\Theta}(t) \rho_{\nu}^{p r o j}(t)$, where $l_{\nu}^{\Theta}(t)$ is a $\nu$ th eigenvalue of $\Theta(t)$ in Liouvillian representation. (2) The Born-Markov assumption and various other types of restrictions for DF behavior does not need to be made, indeed as we show a general approach can be used.

\section{ACKNOWLEDGMENTS}

We gratefully acknowledge financial support through grants from NSERC, MITACS, CIPI, MMO, CITO, and China State Key Projects of Basic Research and Natural Science Foundation (Grant Nos. G1999064509, 79970121, and 60072032).
[1] R. Vrijen, E. Yablonovitch, K. Wang, H. WenJiang, A. Balandin, V. Roychowdhury, T. Mor, and D. DiVincenzo, Phys. Rev. A 62, 012306 (2000).

[2] D. Loss and D.P. DiVincenzo, Phys. Rev. A 57, 120 (1998).

[3] G.P. Berman, G.D. Doolen, and V.I. Tsifrinovich, Superlatt.
Microstruct. 27, 89 (2000).

[4] B.E. Kane, Nature (London) 393, 133 (1998); e-print quant-ph/0003031.

[5] G.P. Berman, G.W. Brown, M.E. Hawley, and V.I. Tsifrinovich, Phys. Rev. Lett. 87, 097902-1 (2001); 86, 2894 
(2001).

[6] C.P. Sun, H. Zhan, and X.F. Liu, Phys. Rev. A 58, 1810 (1998).

[7] D.P. DiVincenzo, J. Appl. Phys. 81, 4602 (1997).

[8] P. Zanardi and M. Rasetti, Mod. Phys. Lett. B 25, 1085 (1997).

[9] P. Zanardi and M. Rasetti, Phys. Rev. Lett. 79, 3306 (1997).

[10] L. Viola, e-print quant-ph/0111167.

[11] E.M. Fortunato, L. Viola, J. Hodges, G. Teklemariam, and D.G. Cory, e-print quant-ph/0111166.

[12] P. Zanardi, e-print quant-ph/0103030.

[13] D.A. Lidar, I.L. Chuang, and K.B. Whaley, Phys. Rev. Lett. 81, 2594 (1998).

[14] D.A. Lidar, D. Bacon, and K.B. Whaley, Phys. Rev. Lett. 82, 4556 (1999).

[15] D. Bacon, J. Kempe, D.A. Lidar, and K.B. Whaley, Phys. Rev. Lett. 85, 1758 (2000).

[16] A. Barenco, A. Berthiaume, D. Deutsch, A. Ekert, R. Jozsa, and C. Macchiavello, SIAM J. Comput. 26, 1541 (1997).

[17] R. Alicki and K. Lendi, Quantum Dynamical Semigroups and Applications (Springer-Verlag, Berlin, 1987).

[18] C. Schön and A. Beige, e-print quant-ph/0104076.

[19] P.G. Kwiat, A.J. Berglund, J.B. Altepeter, and A.G. White,
Science 290, 498 (2000).

[20] D. Kielpinski, V. Meyer, M.A. Rowe, C.A. Sackett, W.M. Itano, C. Monroe, and D.J. Wineland, Science 291, 1013 (2001).

[21] I. Antoniou, Y. Melnikov, and Bi Qiao, Physica A 246, 97 (1997).

[22] T. Petrosky and I. Prigogine, Comput. Math. Appl. 34, 1 (1997).

[23] A. Bohm, H.D. Doebner, and P. Kielanowski, Irreversibility and Causality, Semigroups and Rigged Hilbert Spaces (Springer-Verlag, Berlin, 1998).

[24] M.A. Nielsen and I.L. Chuang, Quantum Computation and Quantum Information (Cambridge University Press, Cambridge, UK, 2000).

[25] G. Burkard, G. Seelig, and D. Loss, Phys. Rev. B 4, 2581 (2000).

[26] G. Burkard and D. Loss, Physica E (Amsterdam) 9, 175 (2001).

[27] J.H. Reina, L. Quiroga, and N.F. Johnson, e-print quant-ph/0105029.

[28] A.O. Caldeira and A.J. Leggett, Phys. Rev. A 31, 1059 (1985). 